\title{
Oxidative Stress in Kawasaki Disease Vasculitis
}

\author{
Kenji Hamaoka*, Tomoyo Yahata, Akiko Okamoto, Chinatsu Suzuki, Yuki Kutsuk, Ayako Yoshioka and Kazuyukiu Ikeda \\ Department of Pediatric Cardiology and Nephrology, Kyoto Prefectural University of Medicine Graduate School of Medical Science, Kyoto, Japan
}

\section{Abstract}

Although the underlying mechanisms by which Kawasaki disease (KD) triggers vasculitis remain unclear, certain stimuli that release inflammatory substances trigger vasculitis during its acute phase, and may continue to cause vascular deterioration in subsequent phases of this disease. Cellular systems responsible for the generation and scavenging of reactive oxygen species (ROS) are clearly involved in the pathogenesis of KD vasculitis. In this manuscript, we discuss studies that illuminate the role of exudative stress in vascular pathology occurring during the acute phase, the interval following the acute phase, and the chronic phase of KD. We also discuss appropriate therapies and the potential role of antioxidant therapy in the treatment and prevention of $\mathrm{KD}$ vasculitis. We conclude there is no clear evidence that current antioxidant therapy can prevent the onset of arteriosclerosis and that administering current antioxidant therapy to children with a prior history of $\mathrm{KD}$ may not immediately improve prognosis. Future prospects should include research on specifically targeted antioxidant treatments and the development of reliable biomarkers for assessing vascular oxidative stress.

\section{Publication History:}

Received: March 24, 2015

Accepted: May 12, 2015

Published: May 14, 2015

\section{Keywords:}

Oxidative stress, Kawasaki disease, Reactive oxygen species, Homeostasis

\section{Introduction}

Kawasaki disease (KD), a condition that occurs most frequently in infants, is characterized by systemic vasculitis of the small and medium arteries [1]. The underlying mechanisms by which $\mathrm{KD}$ triggers vasculitis remain unclear, despite considerable research. However, certain stimuli that generate and release various inflammatory substances within the body, principally infections, are known to trigger vasculitis, causing panangiitis through complex interactions.

When exposed to inflammatory substances, vascular endothelial cells partially contract, forming intercellular gaps. Plasma proteins and other substances that are normally contained within the blood vessels exude from these gaps, forming chemotactic factors. Monocytes and macrophages then infiltrate the site of inflammation, become activated, and produce reactive oxygen species (ROS) [2].

The human body has evolved a system for maintaining redox homeostasis in response to various external stresses. This system begins to scavenge ROS to counteract the production of ROS by the activated immune cells. A healthy individual maintains a proper balance between their ROS generating and scavenging systems. If, however, the balance tilts toward the production of ROS, the excess ROS accumulate, leading to oxidative stress [3,4] (Figure 1). Excess ROS attack proteins, carbohydrates, lipids, nucleic acids, and other constituent substances of the body, resulting in cellular damage that is believed to cause a variety of diseases. In KD specifically, the excess ROS produced by infiltrating inflammatory cells create cytotoxicity that is presumed to play a role in the pathogenesis of vasculitis.

\section{Phlogogenous substances involved in the formation of acute vasculitis}

It is no exaggeration to state that ROS are involved in all proinflammatory pathways. Infiltrating neutrophils and macrophages are the primary sources of the ROS produced during inflammation. Activation of NADH/NADPH oxidase in these inflammatory cells causes rapid and abundant production of ROS that can lead to a state of oxidative stress if not removed by the ROS scavenging system. The arachidonic acid cascade, the activation of xanthine oxidase in vascular endothelial cells [5], or the inhibition of the electron transport system in intracellular mitochondria can also cause overproduction of ROS.

ROS are thought to affect cell function via two mechanisms. The first mechanism involves cellular impairment that occurs through the direct interaction of cellular components with ROS. In the second mechanism, ROS act as second messengers that induce the production of other inflammatory substances, such as tumour necrosis factor (TNF)-a [6-8].

Neutrophil elastase (NE), a substance released from activated neutrophils, is also an important player in the acute inflammatory response [9]. NE is a powerful proteolytic enzyme with low substrate specificity. Infiltration of coronary artery lesions by NE-positive neutrophils has been proven histologically by immunostaining.

The neutrophils, macrophages, and vascular smooth muscle cells that accumulate at the site of inflammation are also known to transiently produce large amounts of nitric oxide (NO) through the action of inducible NO synthase (iNOS) [10]. NO is an unstable radical that forms peroxynitrite (ONOO-) in the presence of excess ROS. NO initially acts as an endothelium-derived relaxing factor (EDRF). Vessels that become resistant to NO constrict when their sensitivity to $\mathrm{NO}$ is lost, exacerbating the effects of inflammation on the dynamics of blood circulation. In addition, ONOO- creates highly reactive radicals that cause significant tissue damage and are thought to induce edema via promotion of vascular permeability.

In summary, the production of various inflammatory substances, including ROS, is positively correlated and highly damaging to tissue.

"Corresponding Author: Dr. Kenji Hamaoka, Department of Pediatric Cardiology and Nephrology, Kyoto Prefectural University of Medicine Graduate School of Medical Science, Kyoto, Japan; E-mail: khamaoka@koto.kpu-m.ac.jp

Citation: Hamaoka K, Yahata T, Okamoto A, Suzuki C, Kutsuk Y, et al. (2015) Oxidative Stress in Kawasaki Disease Vasculitis. Int J Pediatr Neonat Care 1: 103. doi: http://dx.doi.org/10.15344/2455-2364/2015/103

Copyright: (C) 2015 Hamaoka et al. This is an open-access article distributed under the terms of the Creative Commons Attribution License, which permits unrestricted use, distribution, and reproduction in any medium, provided the original author and source are credited. 


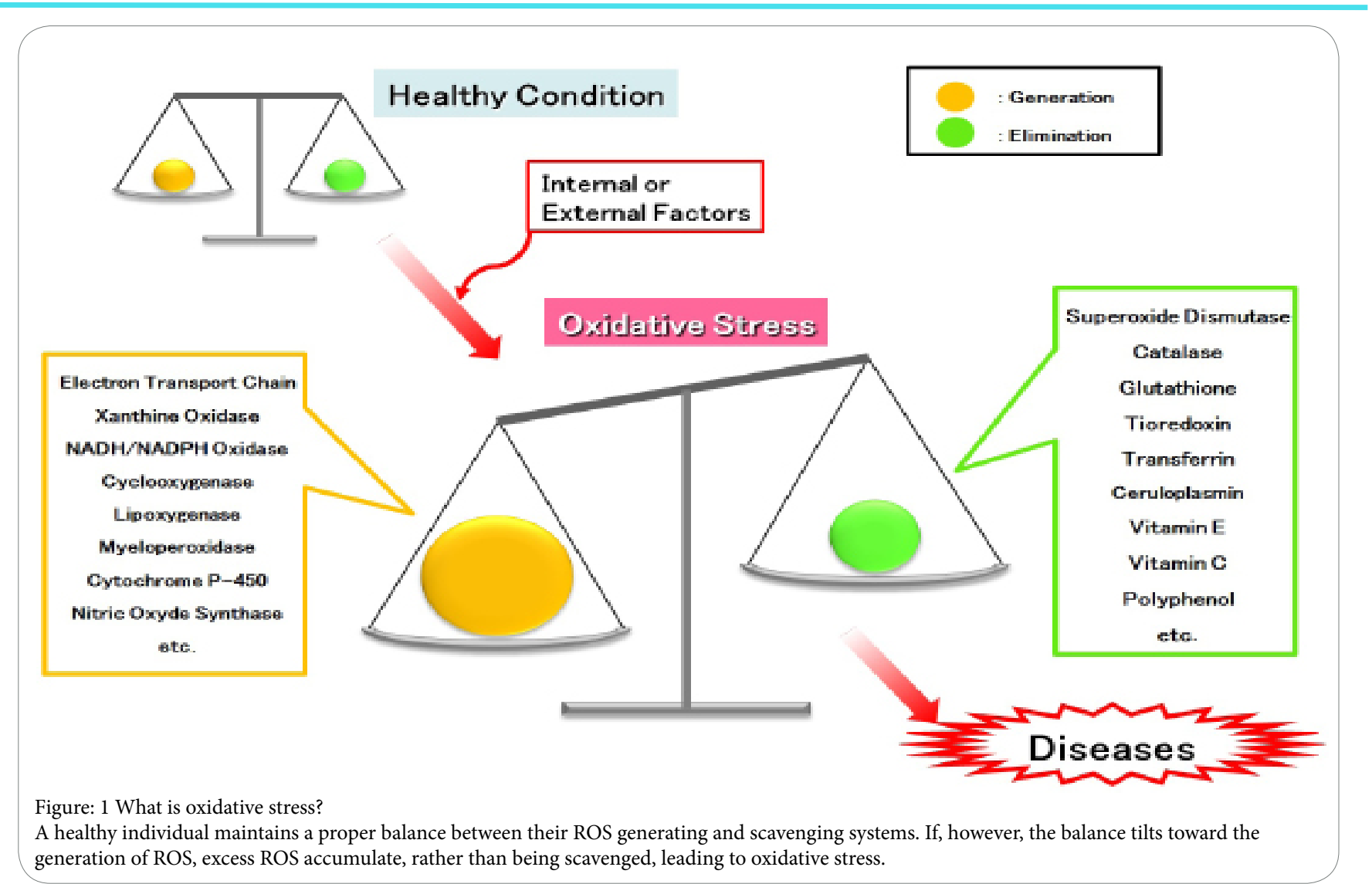

\begin{tabular}{|c|c|c|c|c|c|c|c|}
\hline & & \multicolumn{3}{|c|}{ ROM (U.CARR) } & \multicolumn{3}{|c|}{ BAP (mol/L) } \\
\hline & & Pre-IVIG & Post-IVIG & 2 weeks after & Pre-IVIG & Post-IVIG & 2 weeks after \\
\hline & Median & 633 & 555 & 466 & 2705 & 2714 & 2778 \\
\hline $\begin{array}{l}\text { Unfavorable response } \\
(\mathrm{n}=6)\end{array}$ & $\begin{array}{l}\text { Upper / lower } \\
\text { quartile }\end{array}$ & $687 / 555$ & $646 / 478$ & $506 / 381$ & $2883 / 2510$ & $2940 / 2462$ & $3015 / 2705$ \\
\hline \multirow{3}{*}{$\begin{array}{l}\text { Unfavorable response } \\
(\mathrm{n}=6)\end{array}$} & Median & 564 & 557 & 381 & 2474 & 2438 & 2622 \\
\hline & $\begin{array}{l}\text { Upper / lower } \\
\text { quartile }\end{array}$ & $611 / 531$ & $610 / 483$ & $423 / 304$ & $2557 / 2413$ & $2626 / 2313$ & $2703 / 2380$ \\
\hline & Median & & 312 & & & 2674 & \\
\hline $\begin{array}{l}\text { control } \\
(n=7)\end{array}$ & $\begin{array}{l}\text { Upper / lower } \\
\text { quartile }\end{array}$ & & $327 / 300$ & & & $2745 / 2572$ & \\
\hline
\end{tabular}

Table 1: ROS scavenging system, in patients with acute KD11.

IVIG: intravenous immunoglobulin, ROM: reactive oxygen metabolites, BAP: biological antioxidant potential

These substances are thought to be involved in the etiology of the acute vasculitis associated with $\mathrm{KD}$.

\section{Dynamics of oxidative stress in the acute phase of Kawasaki disease}

We measured the blood reactive oxygen metabolites (ROM) level, which is an index of the activity of the ROS generating system, as well as the serum biological antioxidant potential (BAP), which is an index of the ROS scavenging system, in patients with acute KD [11] (Table $1)$.

The subjects included 19 patients with acute KD (Table 2). Thirteen of these patients responded favourably to the first course of intravenous immunoglobulin (IVIG) treatment $(2 \mathrm{~g} / \mathrm{kg}$ in 1 dose). The remaining 6 patients did not respond favorably to the first course of IVIG. In all cases, blood ROM and BAP levels were measured immediately prior to IVIG treatment, immediately after IVIG treatment ( $24 \mathrm{~h}$ after the end of administration), and 2 weeks after the end of IVIG treatment (Figure 2). In the group of patients who responded poorly to the first round of IVIG, treatment with either additional IVIG or steroids resulted in resolution of fever within 1 week. Although the blood ROM levels were clearly elevated immediately prior to IVIG in the group that responded favorably to treatment, the initial course of IVIG caused a favorable decline. In the group that responded poorly, however, no decline was observed after the initial course of IVIG treatment, even though their ROM levels immediately before IVIG were similar to those in the group that responded favorably. However, there was a significant drop after 2 weeks. No clear fluctuation in BAP levels was observed before or after IVIG treatment in the group of patients who responded favorably 
Citation: Hamaoka K, Yahata T, Okamoto A, Suzuki C, Kutsuk Y, et al. (2015) Oxidative Stress in Kawasaki Disease Vasculitis. Int J Pediatr Neonat Care 1: 103. doi: http://dx.doi.org/10.15344/2455-2364/2015/103

Page 3 of 7

\begin{tabular}{|c|c|c|c|c|c|c|c|c|}
\hline Pt. number & $\begin{array}{l}\text { Responsibility to IVIG } \\
\text { Gender } \\
\text { ( 0: favorable } \\
\text { 1: unfavorable ) }\end{array}$ & $\begin{array}{l}\text { ( } \mathrm{M}: \\
\text { male F: } \\
\text { female ) }\end{array}$ & $\begin{array}{l}\text { Age } \\
\text { (months) }\end{array}$ & $\begin{array}{l}\text { The } \\
\text { beginning } \\
\text { day of } \\
\text { treatments }\end{array}$ & $\begin{array}{l}\text { Coronary } \\
\text { lesions }\end{array}$ & Dilatation & $\begin{array}{l}\text { Additional } \\
\text { treatment }\end{array}$ & $\begin{array}{l}\text { Severity of } \\
\text { illness index ( } \\
\text { Gunma- } \\
\text { score })\end{array}$ \\
\hline 1 & 0 & $\mathrm{~F}$ & 70 & 5 & -- & -- & -- & 0 \\
\hline 2 & 0 & $\mathrm{~F}$ & 22 & 5 & -- & -- & -- & 3 \\
\hline 3 & 0 & $\mathrm{~F}$ & 24 & 4 & -- & -- & -- & 3 \\
\hline 4 & 0 & M & 12 & 4 & -- & -- & -- & 3 \\
\hline 5 & 0 & M & 11 & 5 & -- & -- & -- & 1 \\
\hline 6 & 0 & M & 17 & 4 & -- & -- & -- & Unknown \\
\hline 7 & 0 & M & 18 & 3 & -- & -- & -- & 2 \\
\hline 8 & 0 & M & 26 & 5 & -- & -- & -- & 2 \\
\hline 9 & 0 & M & 14 & 3 & -- & -- & -- & 2 \\
\hline 10 & 0 & $\mathrm{~F}$ & 23 & 6 & -- & -- & -- & 0 \\
\hline 11 & 0 & M & 11 & 3 & -- & -- & -- & 3 \\
\hline 12 & 0 & $\mathrm{~F}$ & 11 & 9 & -- & -- & -- & 1 \\
\hline 13 & 0 & $\mathrm{~F}$ & 39 & 4 & -- & -- & -- & 3 \\
\hline 14 & 1 & $\mathrm{M}$ & 24 & 3 & + & + & IVIG+PSL & 6 \\
\hline 15 & 1 & M & 10 & 4 & -- & -- & IVIG+PSL & 5 \\
\hline 16 & 1 & $\mathrm{~F}$ & 28 & 4 & -- & -- & IVIG+PSL & 6 \\
\hline 17 & 1 & M & 24 & 4 & -- & -- & IVIG+PSL & 6 \\
\hline 18 & 1 & $\mathrm{~F}$ & 36 & 5 & + & + & IVIG+PSL & 6 \\
\hline 19 & 1 & $\mathrm{~F}$ & 10 & 3 & -- & -- & IVIG+PSL & 5 \\
\hline
\end{tabular}

Table 2: 19 patients with acute KD.

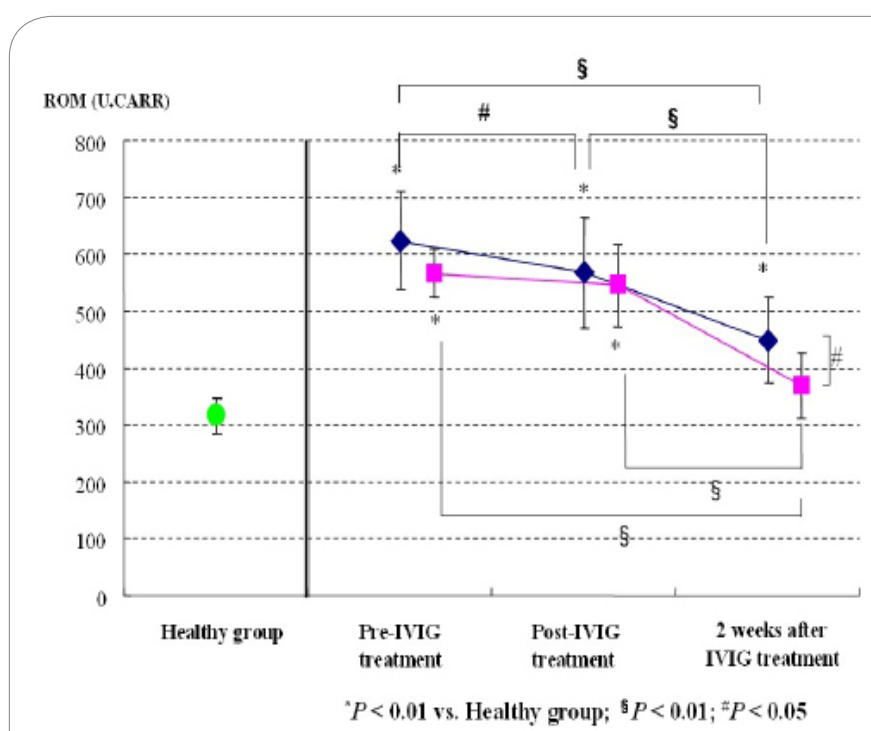

(a) Dynamics of ROM

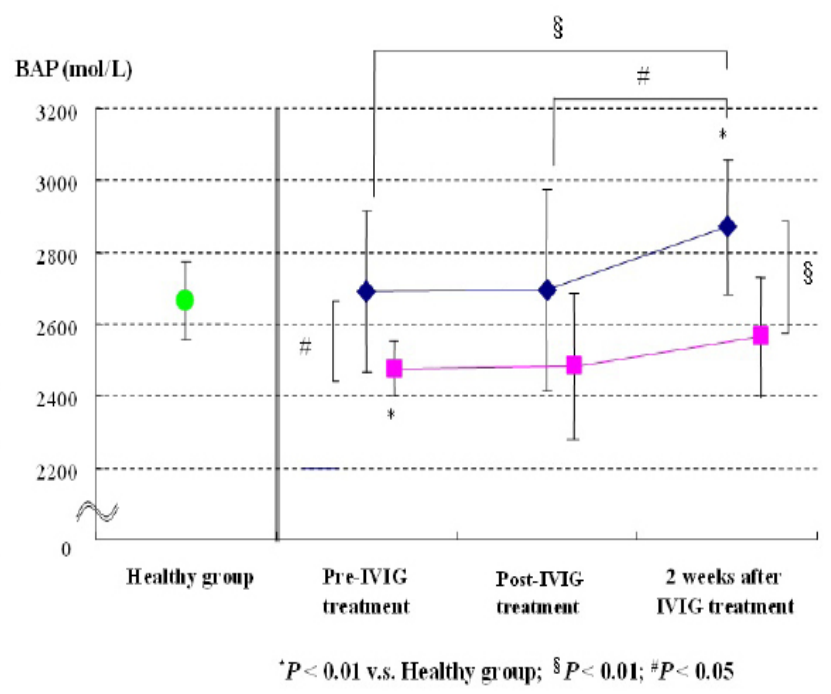

(b) Dynamics of BAP

Figure 2: Dynamics of reactive oxygen metabolites and biological antioxidant potential during the acute stage.

a) Dynamics of reactive oxygen metabolites. Reproduced from [11] with permission from (C) 2011 by the Japanese Circulation Society.

Changes in the levels of reactive oxygen metabolites (ROM) in both treatment groups (blue: favourable, pink: not favourable) in response to intravenous immunoglobulin treatment. Data presented as the mean $\pm \mathrm{SD}$.

b) Dynamics of biological antioxidant potential. Reproduced from [11] with permission from ( 2011 by the Japanese Circulation Society. Changes in the biological antioxidant potential (BAP) of both treatment groups (blue: favourable, pink: not favourable) in response to intravenous immunoglobulin treatment. Data presented as the mean \pm SD. 
to IVIG, but levels tended to gradually increase 2 weeks after IVIG treatment. In the group of patients who responded poorly, however, no significant change in BAP was noted throughout the entire course. The BAP levels immediately before IVIG treatment were significantly lower in the group of patients who responded poorly than in the group that responded favorably $(\mathrm{p}<0.01)$.

These results suggest that ROS generation is significantly enhanced in acute KD, and drops immediately with IVIG treatment. This finding, when used in concert with a reduction of inflammation observed via an independent mechanism, may be useful for determining therapeutic efficacy. In addition, the slight lag in the activation of the ROS scavenging system behind that of the ROS generating system suggests that the ROS scavenging system has a gradual functional increase triggered by the stimulus of increased ROS. However, it is also possible that the capacity of the scavenging system might regulate responsiveness to IVIG treatment and the subsequent ability to recover from the damage sustained during the acute phase.

\section{Future prospects for acute-phase treatments}

Current acute-phase treatments focus on reducing inflammation, and there is a consensus that IVIG is the first line of defense [12]. The results of the recent large-scale RAISE STUDY trial have also brought the efficacy of steroid administration under review [13].

The growing diversity of acute-phase treatments is illustrated by the increasing numbers of clinical reports on the effectiveness of antiTNF- $\alpha$ antibodies, which are not currently indicated for KD $[14,15]$.

Coronary artery involvement is regarded as the most serious complication in acute $\mathrm{KD}$. While the degree of coronary involvement has followed a downward trend in association with the growing diversity of treatments, the problem has not yet been eliminated. The significant role of oxidative stress in the etiology of acute vasculitis, which our research documents, is an important consideration in the development of new treatment strategies. Control of oxidative stress, a factor closely related with inflammation, may quickly alleviate acute inflammation.

\section{Vascular pathology following the acute phase}

Heavily impaired blood vessels do not immediately return to normal, even after the acute phase of KD is successfully overcome, and severe acute inflammation is alleviated. The body's repair mechanisms work slowly to restore original conditions. The time needed for repair is impacted by various conditions, including the extent of damage, the capabilities of the individual's repair mechanism, the presence of repair-inhibiting factors, etc. During this repair period, each patient experiences growth, and some approach adulthood.

At present, there is no consensus concerning the presence or absence of late-stage residual vascular involvement. However, advances in testing methods have revealed evidence of intimal thickening in blood vessels that would have been deemed healthy and normal by conventional testing [16]. In addition, vascular endothelial function testing has recently shown some dysfunction in patients who have a history of KD [17]. There are some reports that the vascular dysfunction may remain to be improved even in chronic phase. Atherosclerotic lesions have also been found in adult KD patients with residual coronary vessel involvement in the chronic phase [18]. Taken together, these reports reflect a growing fear in recent years that $\mathrm{KD}$ vasculitis leads to arteriosclerosis.

\section{Progression of post-inflammation arteriosclerosis or} atherosclerosis and oxidative stress

General explanations for the onset and progression of atherosclerosis include Ross's inflammation response hypothesis [19] and Steinberg's oxidation hypothesis [20]. Namely, when vascular endothelial cells are damaged, various inflammatory cytokines are released. As a result, activated immunocompetent cells adhere to endothelial cells, then migrate below the endothelium. These activated immunocompetent cells are followed by vascular smooth muscle cells, fibroblasts, and other cells. This combined cellular migration results in further endothelial cell damage. Atherosclerotic changes progress as this vicious circle repeats itself.

Attention has recently been focused on the involvement of oxidative stress caused by ROS accumulation in the progression of these atherosclerotic changes. Oxidative stress triggers inflammation by inducing the expression of adhesion factors and chemokines. This induction occurs via NF- $\mathrm{\kappa B}$ activation in vascular endothelial and smooth muscle cells, promoting the formation of atherosclerosis. Superoxides also reduce the expression of vascular endothelial NO synthase (eNOS) and decrease NO production. Decrease NO production triggers contraction of the blood vessel and enhances platelet aggregation, thrombus formation, and intimal proliferation. These factors are believed to exacerbate the progression of vascular endothelial impairment.

\section{Oxidative stress and impaired endothelial function in chronic- phase Kawasaki disease}

To study the relationship between oxidative stress and sustained vascular endothelial cell damage in chronic-phase $\mathrm{KD}$, we measured urine levels of NOx, which are metabolites of nitric oxide, and urine 8-isoprostane levels, a sensitive marker of oxidative stress (Figure 3). The subjects included 149 patients with a history of KD who had reached puberty and a control group of 367 patients who had no coronary risk factors. The 8 -isoprostane levels were notably higher in patients with a history of $\mathrm{KD}$, regardless of whether or not coronary lesions were present. KD patients also showed a significant reduction in NOx, a vasodilation-related factor that originates from vascular endothelial cells. These results suggest the possibility that patients with a history of $\mathrm{KD}$ retain some degree of oxidative stress in the chronic phase, and that this oxidative stress is involved in the appearance and progression of vascular endothelial cell impairment.

Thirty-five patients with a history of KD who had reached adulthood were also assessed for endothelial cell dysfunction (Figure 4). The adults with KD showed a clear drop in the endothelium-dependent vasodilation response (\%FMD) of forearm arteries, compared with 36patients in a control group. These patients also had a clear simultaneous rise in the level of thrombin-antithrombin complex (TAT), a marker of vascular endothelial cell impairment.

\section{Arteroscleroticor atherosclerotic changes in chronic phase}

Chronic-stage vascular endothelial dysfunction and oxidative stress are serious problems for children with a prior history of Kawasaki disease. As these two factors are positively correlated with each other, it is reasonable to hypothesize that they are both involved in the onset and progression of atherosclerosis.

The development of atherosclerosis, also called arteriosclerosis, is a risk factor for coronary events that varies depending on the tissue. 
Citation: Hamaoka K, Yahata T, Okamoto A, Suzuki C, Kutsuk Y, et al. (2015) Oxidative Stress in Kawasaki Disease Vasculitis. Int J Pediatr Neonat Care 1: 103. doi: http://dx.doi.org/10.15344/2455-2364/2015/103

Some question the role of atherosclerosis development in KD. The artherosclerotic changes described in actual late-stage patients have often included so-called post-inflammatory arteriosclerosis, which is rich in hyalinized fibrous tissue.

Previously, using an allergic vasculitis model in weaning rabbits exhibiting KD-like vascular lesions, we reported that the induction of vasculitis led to long-term vascular endothelial cell impairment [21]. In this KD-like model, the additional stress of a high-fat diet during the chronic phase, in which vascular inflammation had already improved, caused the atherosclerosis to worsen. This finding suggests that atherosclerosis is readily induced when hyperlipidemia and other risk factors are combined with vasculitis, which is itself a risk factor for atherosclerosis. That is, when other predisposing conditions linked to increased oxidative stress, such as hypertension, diabetes, hyperlipidemia, obesity, smoking, or stress, coexist, atherosclerosis may have an earlier onset in patients with a prior history of KD [2226] (Figure 5). Oxidative stress not only seems to trigger post-KD vascular impairment, as is the case for many diseases, but also appears to be a factor in deterioration after onset. (a)
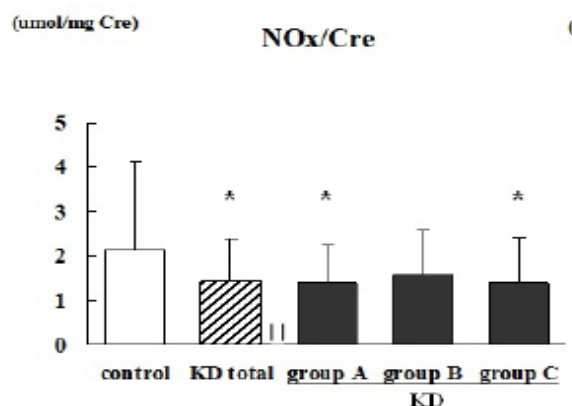

(b)

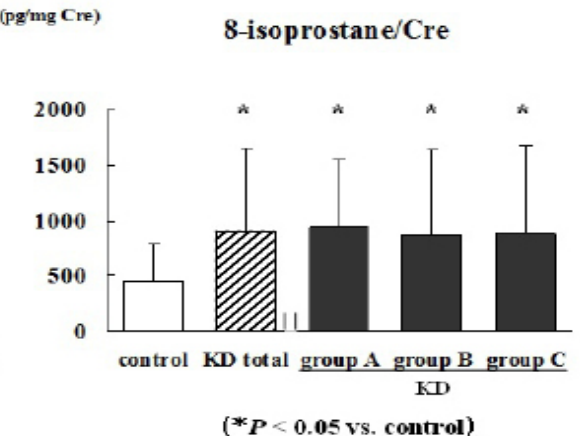

control $n=367$ (male: $n=192$ female: $n=175$ ) : average 15.3 years

KD $n=149$ (male: $n=91$ female: $n=58$ ); average 15.6 years

group $A(n=32)$ : patients with coronary artery lesions (CAL)

group $B(n=21)$ : patients with transient $C A L$

group $C(n=96)$ : patients withont $C A L$

Figure 3: NOx and 8-isoprostane of patients in chronic phase.

Reproduced from [21] with permission from ( 2006 by Ishiyaku Publishers, Inc., Japan. Data are presented as the mean \pm SD. The 8-isoprostane level was notably higher in patients with a history of KD, regardless of whether coronary lesions were present. KD patients also showed a significant reduction in NOx.

(a)

$(\mathbf{n g} / \mathbf{d l})$

\section{TAT}

$P<0.01$

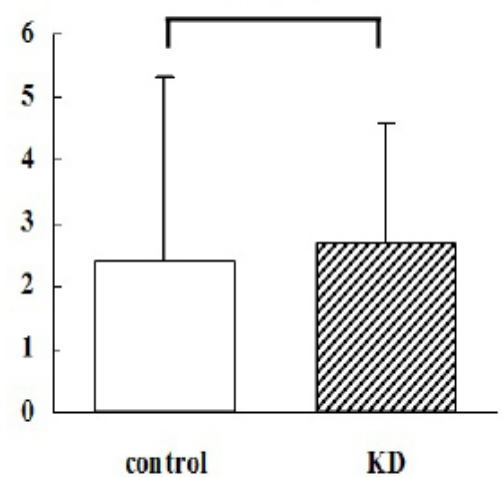

(b)

$(\%)$

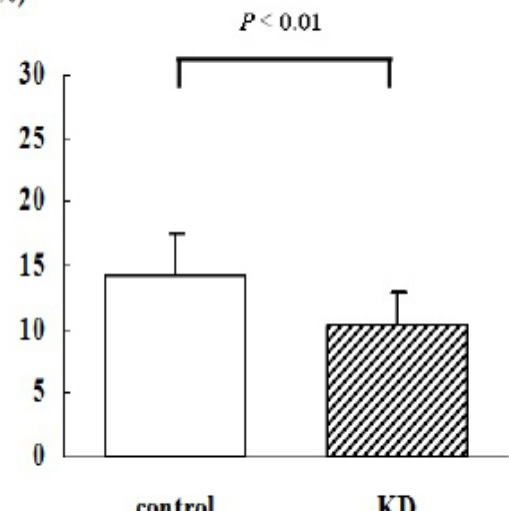

control

Patients with history of KD $: n=35$ ( average 27.0 years )

Controls : $n=36$ ( average 25.5 years)

Figure 4: TAT and \%FMD in chronic-phase patients.

Reproduced from [21] with permission from ๑ 2006 by Ishiyaku Publishers, Inc., Japan.

Data are presented as the mean \pm SD. Adults with KD showed a clear drop in \%FMD compared with 36 patients in a control group. These patients also had a simultaneous clear rise in TAT. 


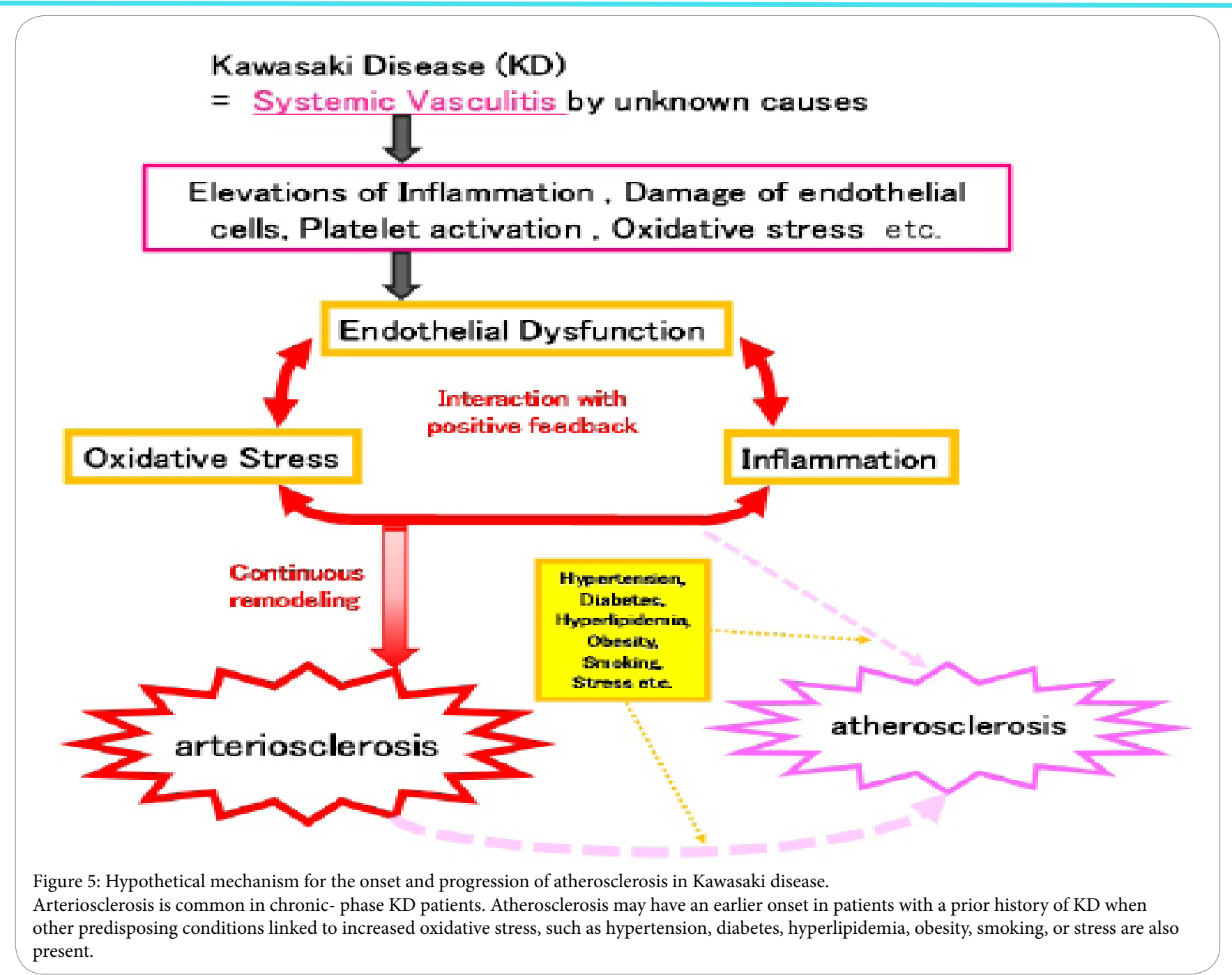

\section{Antioxidant therapy and future prospects}

No clear evidence yet exists that administering antioxidants can mitigate vascular impairment and prevent the onset of artherosclerosis. The incidence of coronary heart disease in France is low despite the high dietary intake of animal fat. This "French paradox" brought attention to the antioxidant action of the polyphenols, which are abundant in red wine [27]. Although subsequent animal research on various antioxidants substantiated their atherosclerosis-inhibiting effect [28], large-scale human clinical trials showed little evidence that they were effective in preventing atherosclerotic disease, and some studies, in fact, showed a tendency toward exacerbation $[29,30]$.

At present, we believe that administering drugs or taking late-stage corrective measures against oxidative stress in children with a prior history of KD may not necessarily immediately improve their vascular prognosis. Despite their cytotoxic effects, ROS play an important role in the biological defense and intracellular signal transduction systems, and their suppression by antioxidant drugs is not necessarily beneficial for the body. Important directions for future research include development of more specifically targeted antioxidant treatment strategies and identification of suitable biomarkers for assessing vascular oxidative stress. Regular examination and early intervention, such as diet and lifestyle guidance, are critical for reducing the risk factors for atherosclerosis in children with a prior history of
$\mathrm{KD}$, particularly as they grow older, in order to improve their vascular prognosis. include development of more specifically targeted antioxidant treatment strategies and identification of suitable biomarkers for assessing vascular oxidative stress. Regular examination and early intervention, such as diet and lifestyle guidance, are critical for reducing the risk factors for atherosclerosis in children with a prior history of $\mathrm{KD}$, particularly as they grow older, in order to improve their vascular prognosis.

\section{Competing Interests}

The authors declare that they have no competing interests exit.

\section{Author Contributions}

All the authors substantially contributed to the study conception and design as well as the acquisition and interpretation of the data and drafting the manuscript.

\section{References}

1. Kawasaki T (1967) Acute febrile mucocutaneous lymph node syndrome in young children with unique digital desquamation. Allergy. 16: 178-222.

2. Cave AC, Brewer AC, Narayanapanicker A, Ray R, Grieve DJ, et al. (2006) $\mathrm{NADPH}$ oxidases in cardiovascular health and disease. Antioxid Redox Signal 8: 691-728. 
Citation: Hamaoka K, Yahata T, Okamoto A, Suzuki C, Kutsuk Y, et al. (2015) Oxidative Stress in Kawasaki Disease Vasculitis. Int J Pediatr Neonat Care 1: 103. doi: http://dx.doi.org/10.15344/2455-2364/2015/103

Page 7 of 7

3. Sies H (1985) Oxidative stress: inductor remarks. In: Sies H (ed) Oxidative stress. Academic Press, London 1-8.

4. Sies H (2000) What is oxidative stress? In: Kency JF Jr. (ed) Oxidative stress and vascular disease. Kluwer Academic Publishers 1-8.

5. Phan SH, Gannon DE, Varani J, Ryan US, Ward PA (1989) Xanthine oxidase activity in rat pulmonary artery endothelial cells and its alteration by activated neutrophils. Am J Pathol 134: 1201-1211.

6. Furukawa S, Matsubara T, Jujoh K, Yone K, Sugawara T, et al. (1988) Peripheral blood monocyte/macrophages and serum tumor necrosis factor in Kawasaki disease. Clin Immunol Immunopathol 48: 247-251.

7. Furukawa S, Matsubara T, Yone K, Hirano Y, Okumura K, et al. (1992) Kawasaki disease differs from anaphylactoid purpura and measles with regard to tumour necrosis factor-alpha and interleukin 6 in serum. Eur Pediatr 151: 44-47.

8. Lin CY, Lin CC, Hwang B, Chiang B (1992) Serial changes of serum interleukin-6, interleukin-8, and tumour necrosis factor alpha among patients with Kawasaki disease. J Pediatr 21: 924-926.

9. Inamo Y, Harada K, Okuni M, Kimoto K, Takeuchi S, et al. (1987) Immunoreactive polymorphonuclear leukocyte elastase in complex with alpha 1-antitrypsin in Kawasaki disease. Acta Paediatr Jpn 29: 202-205.

10. Buttery LDK, Springall DR, Chester AH, Evans TJ, Standfield N, et al. (1996) Inducible nitric oxide synthase is present within human atherosclerotic lesions and promotes the formation and activity of peroxynitrite. Lab Invest 75: 77-785.

11. Yahata T, Suzuki C, Hamaoka A, Fujii M, Hamaoka K (2011) Dynamics of reactive oxygen metabolites and biological antioxidant potential in the acute stage of Kawasaki disease. Circ J 75: 2453-2459.

12. JCS Joint Working Group (2010) Guidelines for diagnosis and management of cardiovascular sequelae in Kawasaki disease (JCS 2008)--digest version. Circ J 74: 1989-2020.

13. Kobayashi T, Saji T, Otani T, Takeuchi K, Nakamura T, et al. RAISE study group investigators (2012) Efficacy of immunoglobulin plus prednisolone for prevention of coronary artery abnormalities in severe Kawasaki disease (RAISE study): a randomized, open-label, blinded-endpoints trial. Lance 28: $1613-1620$

14. Weiss JE, Eberhard BA, Chowdhury D, Gottlieb BS (2004) Infliximab as a novel therapy for refractory Kawasaki disease. J Rheumatol 31: 808810. Burns JC, Mason WH, Hauger SB, Janai H, Bastian JF, et al. (2005) Infliximab treatment for refractory Kawasaki syndrome. J Pediatr 146: 662667

15. Sugimura T, Kato H, Inoue O, Fukuda T, Sato N, et al. (1994) Intravascular ultrasound of coronary arteries in children. Assessment of the wall morphology and the lumen after Kawasaki disease. Circulation 89: 258265.

16. Niboshi A, Hamaoka K, Sakata K, Yamaguchi N (2008) Endothelial dysfunction in adult patients with a history of Kawasaki disease. Eur $J$ Pediatr 167: 189-196.

17. Takahashi K, Oharaseki T, Naoe S (2001) Pathological study of postcoronary arteritis in adolescents and young adults: with reference to the relationship between sequelae of Kawasaki disease and atherosclerosis. Pediatr Cardiol 22: 138-142

18. Ross R (1999) Atherosclerosis--an inflammatory disease. N Engl J Med 340: $115-126$

19. Steinberg D, Parthasarathy S, Carew TE, Khoo JC, Witztum JL (1989) Beyond cholesterol. Modifications of low-density lipoprotein that increase its atherogenicity. N Engl J Med 320: 915-924.

20. Liu Y, Onouchi Z, Sakata K, Ikuta K (1996) An experimental study on the role of smooth muscle cells in the pathogenesis of atherosclerosis of the coronary arteritis. J Japan Pediatr Soc 100: 1453-1458.

21. Silva BR, Pernomian L, Bendhack LM (2012) Contribution of oxidative stress to endothelial dysfunction in hypertension. Front Physiol 3: 441
22. Silva BR, Pernomian L, Bendhack LM (2012) Contribution of oxidative stress to endothelial dysfunction in hypertension. Front Physiol 3: 441.

23. Stephens JW, Khanolkar MP, Bain SC (2009) The biological relevance and measurement of plasma markers of oxidative stress in diabetes and cardiovascular disease. Atherosclerosis 202: 321-329.

24. Araujo FB, Barbosa DS, Hsin CY, Maranhão RC, Abdalla DS (1995) Evaluation of oxidative stress in patients with hyperlipidemia. Atherosclerosis 117: 61-71.

25. Furukawa S, Fujita T, Shimabukuro M, Iwaki M, Yamada Y, et al. (2004) Increased oxidative stress in obesity and its impact on metabolic syndrome. J Clin Invest 114: 1752-1761.

26. Morrow JD, Frei B, Longmire AW, Gaziano JM, Lynch SM, et al. (1995) Increase in circulating products of lipid peroxidation (F2-isoprostanes) in smokers. Smoking as a cause of oxidative damage. N Engl J Med 332: 1198-1203

27. Ferrières $\mathrm{J}$ (2004) The French paradox: lessons for other countries. Heart 90: 107-111.

28. Praticò D, Tangirala RK, Rader DJ, Rokach J, FitzGerald GA (1998) Vitamin $E$ suppresses isoprostane generation in vivo and reduces atherosclerosis in ApoE-deficient mice. Nat Med 4: 1189-1192.

29. Bjelakovic G, Nikolova D, Gluud LL, Simonetti RG, Gluud C (2007) Mortality in randomized trials of antioxidant supplements for primary and secondary prevention: systematic review and meta-analysis. JAMA 297: 842-857.

30. Baillie JK, Thompson AA, Irving JB, Bates MG, Sutherland Al, et al. (2009) Oral antioxidant supplementation does not prevent acute mountain sickness: double blind, randomized placebo-controlled trial. QJM 102: 341348. 